University of Wollongong

Research Online

Australian Institute for Innovative Materials -

Papers

Australian Institute for Innovative Materials

$1-1-2018$

Coordination of Atomic Co-Pt Coupling Species at Carbon Defects as Active Sites for Oxygen Reduction Reaction

Longzhou Zhang

Griffith University

Julia Fischer

University of Queensland

Yi Jia

Griffith University

Xuecheng Yan

Griffith University

Wei Xu

Chinese Academy Of Sciences, Jilin University

See next page for additional authors

Follow this and additional works at: https://ro.uow.edu.au/aiimpapers

Part of the Engineering Commons, and the Physical Sciences and Mathematics Commons

Research Online is the open access institutional repository for the University of Wollongong. For further information contact the UOW Library: research-pubs@uow.edu.au 


\title{
Coordination of Atomic Co-Pt Coupling Species at Carbon Defects as Active Sites for Oxygen Reduction Reaction
}

\author{
Abstract \\ Platinum (Pt) is the state-of-the-art catalyst for oxygen reduction reaction (ORR), but its high cost and \\ scarcity limit its large-scale use. However, if the usage of Pt reduces to a sufficiently low level, this critical \\ barrier may be overcome. Atomically dispersed metal catalysts with high activity and high atom efficiency \\ have the possibility to achieve this goal. Herein, we report a locally distributed atomic Pt-Co nitrogen- \\ carbon-based catalyst (denoted as A-CoPt-NC) with high activity and robust durability for ORR (267 times \\ higher than commercial Pt/C in mass activity). The A-CoPt-NC shows a high selectivity for the 4e-pathway \\ in ORR, differing from the reported 2e-pathway characteristic of atomic Pt catalysts. Density functional \\ theory calculations suggest that this high activity originates from the synergistic effect of atomic Pt-Co \\ located on a defected $\mathrm{C} / \mathrm{N}$ graphene surface. The mechanism is thought to arise from asymmetry in the \\ electron distribution around the $\mathrm{Pt} / \mathrm{Co}$ metal centers, as well as the metal atoms' coordination with local \\ environments on the carbon surface. This coordination results from N8V4 vacancies (where N8 \\ represents the number of nitrogen atoms and V4 indicates the number of vacant carbon atoms) within \\ the carbon shell, which enhances the oxygen reduction reaction via the so-called synergistic effect. \\ Disciplines \\ Engineering | Physical Sciences and Mathematics

\section{Publication Details} \\ Zhang, L., Fischer, J. Melisande Theresa Agatha., Jia, Y., Yan, X., Xu, W., Wang, X., Chen, J., Yang, D., Liu, H., \\ Zhuang, L., Hankel, M., Searles, D. J., Huang, K., Feng, S., Brown, C. L. \& Yao, X. (2018). Coordination of \\ Atomic Co-Pt Coupling Species at Carbon Defects as Active Sites for Oxygen Reduction Reaction. Journal \\ of the American Chemical Society, 140 (34), 10757-10763.

\section{Authors} \\ Longzhou Zhang, Julia Fischer, Yi Jia, Xuecheng Yan, Wei Xu, Xiyang Wang, Jun Chen, Dongjiang Yang, \\ Hongwei Liu, Linzhou Zhuang, Marlies Hankel, Debra J. Searles, Keke Huang, Shouhua Feng, Christopher \\ L. Brown, and Xiangdong Yao
}




\section{Coordination of atomic Co-Pt coupling species at carbon defects as electrochemical Janus active sites}

Longzhou Zhang $^{1^{\perp}}$, Julia Melisande Theresa Agatha Fischer ${ }^{\perp}$, Yi Jia ${ }^{1 \star}$, Xuecheng Yan ${ }^{1}$, Wei Xu ${ }^{3}$, Xiyang Wang ${ }^{4}$, Jun Chen ${ }^{5}$, Dongjiang Yang ${ }^{1}$, Hongwei Liu ${ }^{6}$, Marlies Hankel ${ }^{2}$, Debra J. Searles ${ }^{2,7}$, Keke Huang ${ }^{4}$, Shouhua Feng ${ }^{4}$, Christopher L Brown ${ }^{1}$ and Xiangdong $\mathrm{Yao}^{1,4 \star}$

${ }^{1}$ School of Natural Sciences and Queensland Micro- and Nanotechnology Centre, Griffith University, Nathan Campus, Queensland 4111, Australia.

${ }^{2}$ Centre for Theoretical and Computational Molecular Science, Australian Institute for Bioengineering and Nanotechnology, The University of Queensland, Queensland 4072, Australia.

${ }^{3}$ Beijing Synchrotron Radiation Facility, Institute of High Energy Physics, Chinese Academy of Sciences, Beijing 100049, P. R. China.

${ }^{4}$ State Key Laboratory of Inorganic Synthesis and Preparative Chemistry, College of Chemistry, Jilin University, Changchun 130012, P. R. China.

${ }^{5}$ Intelligent Polymer Research Institute, ARC Centre of Excellence for Electromaterials Science, AIIM Facility, University of Wollongong, Innovation Campus, Wollongong, New South Wales 2522, Australia.

${ }^{6}$ Australian Centre for Microscopy \& Microanalysis (ACMM), the University of Sydney, Sydney, NSW 2006, Australia.

${ }^{7}$ School of Chemistry and Molecular Biosciences, The University of Queensland, Queensland 4072, Australia.

E-mail: y.jia@griffith.edu.au and x.yao@griffith.edu.au

${ }^{\perp}$ L. Z. Zhang and J. M. T. A. Fischer contributed equally. 


\section{Abstract}

Platinum (Pt) is the state-of-the-art catalyst for oxygen reduction (ORR) and hydrogen evolution (HER), but its high cost and scarcity limit its large-scale use. However, if the usage of Pt can be reduced to a sufficiently low level $\left(0.125 \mathrm{mg} / \mathrm{cm}^{2}\right.$ according to the Department of Energy (DOE) target), this critical barrier may be overcome. Atomically dispersed metal catalysts with high activity and high atom efficiency make them possible to achieve this goal. Herein, we report a locally distributed atomic Pt-Co nitrogen-carbon based catalyst (denoted as A-CoPt-NC) with high activity and robust durability for ORR (267 times higher than commercial Pt/C in mass activity) and HER (much superior to Pt/C). The A-CoPt-NC shows a high selectivity for the $4 \mathrm{e}^{-}$pathway in ORR, differing from the reported $2 \mathrm{e}^{-}$pathway characteristic of atomic Pt catalysts. Importantly, the A-CoPt-NC catalyst in this experiment only contains $\sim 0.0005 \mathrm{mg} / \mathrm{cm}^{2}$ of Pt and the activity is about 20 times the DOE target at $0.9 \mathrm{~V}$ in alkaline solution. Density functional theory (DFT) calculations suggest that this high activity originates from the synergistic effect of atomic Pt-Co located on a defected $\mathrm{C} / \mathrm{N}$ graphene surface. The mechanism is thought to arise from asymmetry in the electron distribution around the Pt/Co metal centres, as well as the metal atoms coordination with local environments on the carbon surface. This coordination results from N8V4 vacancies (where N8 represents the number of nitrogen atoms, V4 indicates the number of vacant carbon atoms) within the carbon shell that enhances the oxygen reduction reaction via the so-called synergistic effect. DFT calculations suggest the HER activity arises from atomic Pt-Co coupling species locating at N6V4 vacancies within the graphitic shell causing the A-CoPt-NC catalyst to show extremely high HER activities in both acid and alkaline solutions. 
Platinum $(\mathrm{Pt})$ is the benchmark electrocatalyst for the oxygen reduction reaction $(\mathrm{ORR})^{1-5}$ and hydrogen evolution reaction (HER $)^{6,7}$, exhibiting high activities. However, the high cost and the natural scarcity of Pt still hamper its industrial implementation. Downsizing the Pt particles to expose more $\mathrm{Pt}$ atoms on the surface (rendering higher atom efficiency) is a viable strategy to enable Pt based catalysts more affordable. Generally, when particle sizes are further reduced to the nanoscale, quantum size effects will be induced in the catalysts, which not only alter the surface energy due to the unsaturated coordination, but change the $d$ state energy of metal atoms leading to spatial electron localization ${ }^{8,9}$. This size-induced change of electronic structures at active sites will subsequently tailor the binding capability with the diverse species of reactants (e.g. $\mathrm{O}_{2}$ in ORR or $\mathrm{H}^{+}$in HER), thus increases in the activities of catalysts of the electrocatalytic reactions are attainable.

Recently, the so-called single-atom catalyst (SACs) have sparked new interests in heterogeneous catalysis, maximizing the atom efficiency and demonstrating excellent catalytic performance in $\mathrm{CO}$ oxidation ${ }^{10-12}$, water-gas shift (WGS) reaction ${ }^{13},{ }^{14}$, and electrochemical/photoelectrochemical reactions ${ }^{15-17}$. However, the development of SACs in electrocatalysis is still in its infancy, as several issues are urgently to be addressed. Firstly, SACs are not really "atoms" (i.e. zero valence state), as the atomic metal species interact strongly with the neighbouring atoms on support and exhibit valence states. This pivotal fact pushes us to reconsider that the active sites in SACs are originated from the unique coordination structures between the single metal atoms and surround non-metallic atoms of the support. Secondly, optimization of the electronic structure on the active sites by modulating the coordination environment of the metal atoms (MAs) has become a critical route to enhance the reactivity of the active sites. Take the MA-N-C $(\mathrm{MA}=\mathrm{Fe}$ or $\mathrm{Co})$ coordination structure for an example, the MA-N $\mathrm{N}_{2}$ moieties as active centres have been reported more efficient for ORR than those of the MA- $\mathrm{N}_{4}$ moieties. This is ascribed to the more suitable interaction of MA- $\mathrm{N}_{2}$ 
moieties with $* \mathrm{O}_{2}$ and $* \mathrm{OH}$ intermediates ${ }^{15,18}$. Thirdly, selectivity in SACs for specific electrochemical reactions is important. For instance, the isolated atomic Pt species (denoted as IA-Pt) were reported to possess a high selectivity for the production of $\mathrm{H}_{2} \mathrm{O}_{2}$ via a $2 \mathrm{e}^{-}$pathway $\left(\mathrm{O}_{2}+2 \mathrm{H}^{+}+2 e^{-} \rightarrow \mathrm{H}_{2} \mathrm{O}_{2}\right)$ rather than $\mathrm{H}_{2} \mathrm{O}$ via a $4 \mathrm{e}^{-}$pathway $\left(\mathrm{O}_{2}+4 \mathrm{H}^{+}+4 e^{-} \rightarrow 2 \mathrm{H}_{2} \mathrm{O}\right)$. This is because the breaking of the $\mathrm{O}-\mathrm{O}$ bond is not energetically favourable on the isolated atomic Pt sites ${ }^{19-21}$. However, it is possible to alter the reaction to a $4 \mathrm{e}^{-}$pathway for ORR by putting another Pt (or different metal) atom at a certain distance. Fourthly, the research on the atomic interaction between metallic atoms in a certain local environment may provide insightful understanding of "synergetic effect". The so-called "synergetic effect" has been extensively used, but it is very rare to studies on its origination, especially at the atomic level. Therefore, it is imperative to get an in-depth insight into the interactions between the atomic metal species and local environment on the support (e.g. atomic metal-nonmetal coordination), which may direct us to design the new generation of atomic metal catalysts with high activity and selectivity. Very recently, our group developed a defective graphene (DG) with a high density of structural defects ${ }^{22}$. Besides the defects themselves activating the electrochemical reactions according to the proposed defect mechanism ${ }^{22}$, defects are highly likely to provide unique sites for trapping metallic species ${ }^{23}, 24$. Due to the different structures and sizes of the defects, one or more metal atoms might be trapped into one specific defect, providing the possibility of studying the interaction between the single metal atom and neighboring nonmetal atoms, the interaction between the metal atom pairs (in this case atomic $\mathrm{Pt}-\mathrm{Me}(\mathrm{Me}=\mathrm{Co} / \mathrm{Pt})$ coupling species) and the interaction between the metal atom pair and the neighboring nonmetal atoms. Additionally, compared to the metal oxide supports, defective carbon presents a high tolerance in electrolyte environments at a wide range of $\mathrm{pH}$, which enables the catalyst to work in alkaline, neutral and acid electrolytes. This is particularly relevant to the HER in this study. 
Herein, we report a new class of atomic Co-Pt carbon/ $\mathrm{N}$ based catalyst (denoted as ACoPt-NC) that directly utilized the induced defects in the shell of carbon capsules to form atomic Co-Pt-N-C coordination structures as active sites through electrochemical activation. According to the analysis of X-ray absorption near-edge structure (XANES), the atomic configurations between $\mathrm{Co} / \mathrm{Pt}$ and N/C can be deduced. Direct observation from HAADFSTEM image clearly demonstrates that the atomic metals $(\mathrm{Co} / \mathrm{Pt})$ are trapped into a vacancy type defect to form integrity of atomic Co-Pt-N-C coordination structures. Experimentally, the obtained A-CoPt-NC catalyst exhibited very high activity and robust stability for the ORR in alkaline solution, delivering the specific (electrochemical active surface area (ECSA) normalized to Pt mass) and mass activities of 85 and 267 times greater than those of the commercial Pt/C catalyst, respectively. Meanwhile, the activity had no obvious loss after a 240 min electrochemical durability test. The atomic Pt shows high selectivity for the $4 \mathrm{e}^{-}$pathway in ORR, which is different from the counterpart reported in former literatures ${ }^{19-21}$. Furthermore, A-CoPt-NC exhibits extremely high activities in HER under all $\mathrm{pH}$ (acidic, neutral and alkaline) conditions. Density functional theory (DFT) calculations on model structures developed based on the observed and other possible $\mathrm{Co}-\mathrm{Pt}-\mathrm{N}-\mathrm{C}$ configurations reveal that atomic Pt-Me $(\mathrm{Me}=\mathrm{Co} / \mathrm{Pt}$ ) coupling at the carbon defects (denoted as a(Pt-Me)@NXVY where $\mathrm{X}$ is the number of nitrogens surrounding the defect and $\mathrm{Y}$ is the number of carbon atoms removed) can significantly tailor the electronic structure of the metal atoms and alter the charge distribution at the coordination structures, thereby enhancing the specific electrocatalytic performances (a(Pt-Co)@N8V4 for ORR and a(Pt-Co)@N6V4 for HER).

\section{Preparation and characterization of A-CoPt-NC electrocatalyst}



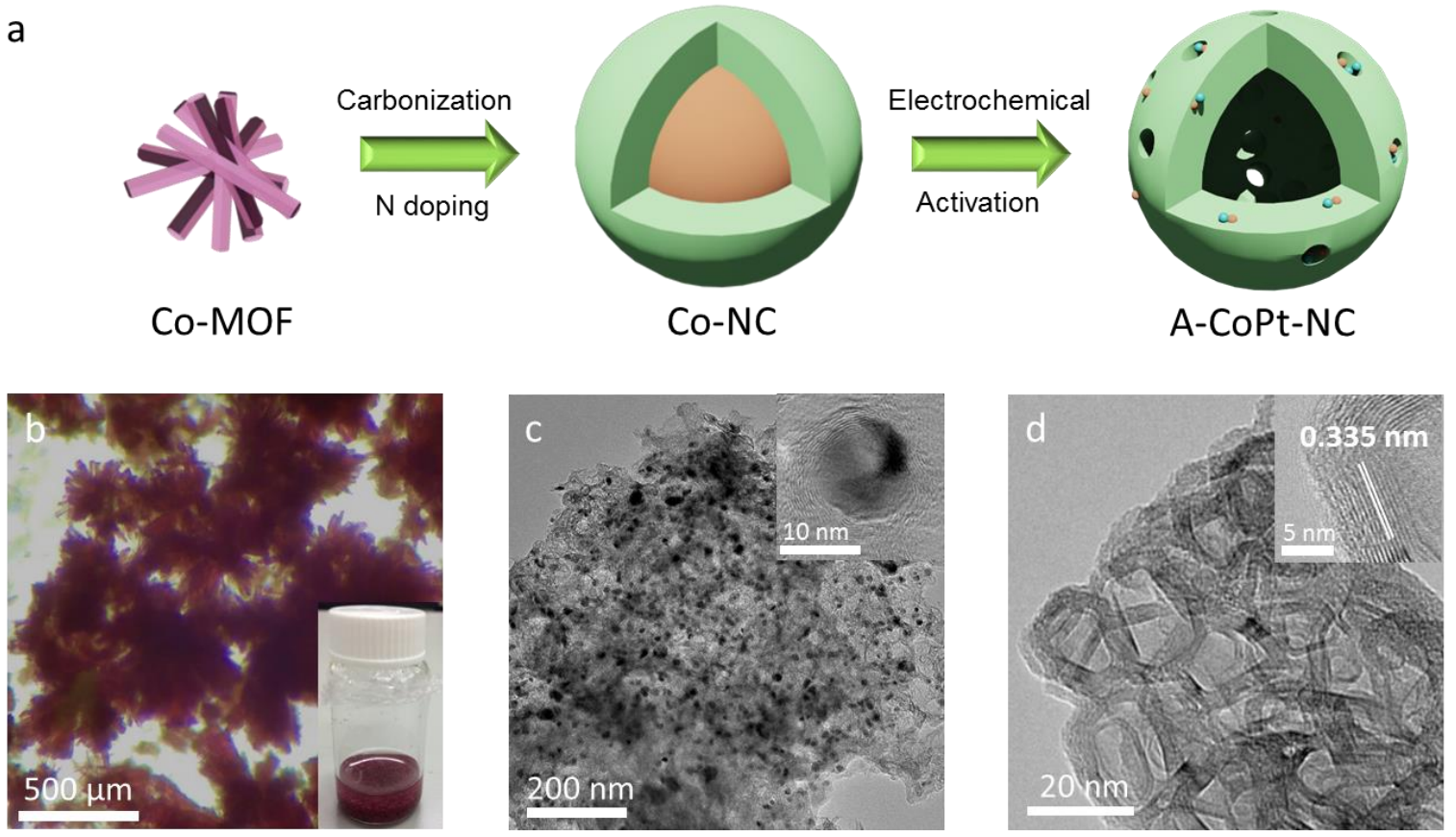

Figure 1 | Preparation and morphology characterization of A-CoPt-NC. a, Schematic illustration of the synthesis procedure of A-CoPt-NC. $\mathbf{b}$, An optical photo of the Co-MOF crystal. The inset image shows Co-MOF in the DMF solution. c, TEM images of the Co-NC composite. The inset TEM image demonstrates the core-shell structure. $\mathbf{d}$, TEM images of A-CoPt-NC. The inset TEM image demonstrates the hollow graphitic shells.

A-CoPt-NC was fabricated by a facile two-step synthesis strategy with the precursor of rodlike cobalt-metal organic framework (Co-MOF) as shown in Fig. 1a. In the first step, the asprepared Co-MOF (Fig. $1 \mathrm{~b}$ and inset) was carbonized at $850{ }^{\circ} \mathrm{C}$ with simultaneous nitrogen doping treatment, forming the core-shell Co-NC structure (Fig. 1c and inset). We then electrochemically applied a cyclic-potential to the Co-NC electrode (a similar activation process can be referred to the literature ${ }^{25,26}$ ). Thereby carbon based hollow nanostructures with graphitic shells were generated (Fig. 1d and inset). Although XRD patterns show that no obvious metallic crystal phases exist after the electrochemical activation (Supplementary 
Fig.1), the characterization of both inductively coupled plasma atomic emission spectroscopy (ICP-AES) and energy-dispersive X-ray spectroscopy (EDS) elemental mapping (Supplementary Fig.2) demonstrate trace amounts of Co and Pt in A-PtCo-NC (the content of Co is $1.72 \mathrm{wt} \%$, compared to $21.10 \mathrm{wt} \%$ before activation, and the content of $\mathrm{Pt}$ is $0.16 \mathrm{wt} \%$ ), indicating almost all of the Co cores has been removed. These results prompted us to propose that the atomically disperse $\mathrm{Co}$ and $\mathrm{Pt}$ co-doped in $\mathrm{NC}$ capsules can form during the electrochemical activation process, which will be verified below.
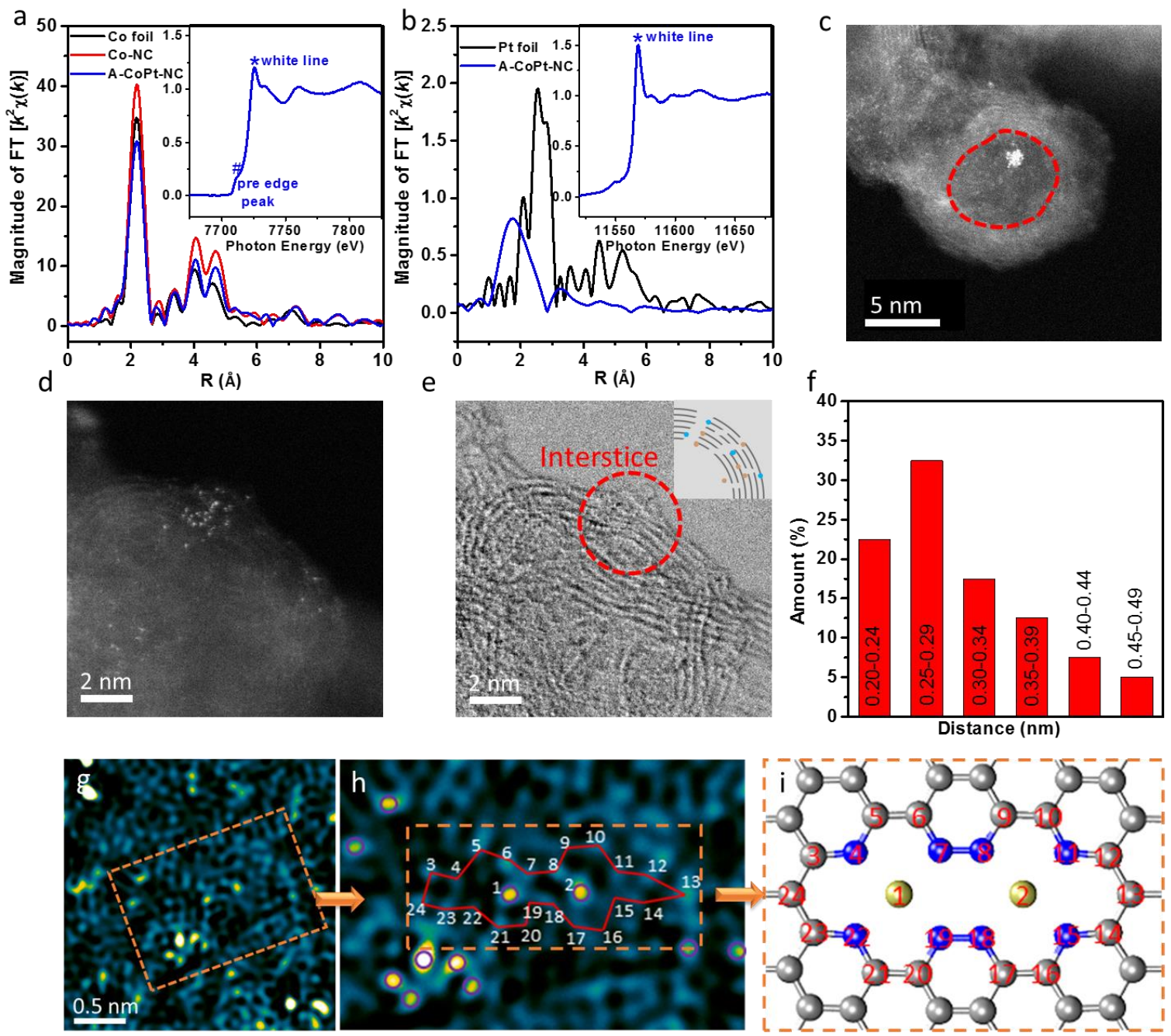

Figure 2 | The structural characterizations of A-CoPt-NC. a,b, The $k^{2}$-weighted Fourier transform spectra of the Co and Pt EXAFS for Co-NC, A-CoPt-NC, Co foil and Pt foil, respectively. The insets are the Co and Pt XANES spectra, respectively, for A- 
CoPt-NC. c,d, The dark-field STEM images of the A-CoPt-NC. The position of the cobalt core dissolved in the activation, is marked with the red dashed ring. e, The corresponding bright-field STEM image of (d) with the inset of the schematic diagram of the interstice zone. $\mathbf{f}$, The distribution of distances of adjacent metal atoms counted from 40 neighbouring metal atom pairs. $\mathbf{g}$, The HAADF image of A-CoPt-NC after fast Fourier Transformation (FFT) filtering. The bright yellow spots are metal atoms and the cyan spots are carbon atoms. $\mathbf{h}$, A partially zoomed-in image of the area framed in $\mathbf{g}$. Metal atoms are marked by purple circles. The carbon atoms adjacent to \#1 and \#2 metal atoms are linked with red line. $\mathbf{i}$, Model of the configuration of the 2 metal atoms trapped in the defect, reconstructed from the observed atomic structure in $\mathbf{h}$.

To further investigate the fine structure of Pt and Co on carbon, we performed X-ray absorption near-edge structure (XANES) and extended X-ray absorption fine structure (EXAFS) spectrometry. The intensity of Co-Co peak around $2.2 \AA$ decays after the activation, revealing a change in the Co local environment (Fig. 2a) $)^{15,27}$. The dissolution of Co cores during activation disrupts the majority of the original Co-Co coordination and the residual Co clusters contribute to the declined Co-Co peak (there may also exist some undissolved Co cores although we did not observe any in searching domain by TEM). This is further confirmed by the aberration-corrected scanning transmission electron microscopy (STEM) images. In Fig. $2 \mathrm{c}$, the area of the initial Co core before activation is marked with a red dashed ring. It can be seen that the Co core was removed and only a small Co cluster sized $\sim 1 \mathrm{~nm}$ remained, which agrees with the EXAFS analysis. The high intensity of the white line of A-CoPt-NC in the XANES (inset of Fig.2a) indicates an oxidized electronic structure of Co, which is due to the $\mathrm{Co}-\mathrm{N}$ and/or $\mathrm{Co}-\mathrm{C}$ and/or Co-C/N coordination newly formed during the activation process ${ }^{15}$. It is suggested that the pre-edge peak of A-CoPt-NC at $7712 \mathrm{eV}$ is the fingerprint of the $\mathrm{Co}-\mathrm{N}_{4}$ square planar structure ${ }^{28,29}$ due to the dipole forbidden $1 \mathrm{~s} \rightarrow 3 \mathrm{~d}$ transition with dominantly 
quadrupole coupling, which is analogous to the pre-edge peak of cobalt phthalocyanine ${ }^{30}$. This finding is also consistent with the previous report of atomic Co catalyst used for HER ${ }^{27}$. It is very difficult to distinguish the bond of the $\mathrm{Co}-\mathrm{N}_{4}$ and $\mathrm{Co}-\mathrm{C}_{4}$ due to the very small difference of the bonding energy. However, it is reasonable to suppose that the coordination is Co-C/N 4 because of the large amount of $\mathrm{N}$ in carbon shell.

In the $\mathrm{R}$ space spectra of Pt (Fig. 2b), the scattering peak derived from Pt-Pt coordination at $2.6 \AA$ is not observed, in contrast to the Pt foil, indicating atomic dispersion of Pt species in the N-doped carbon capsules. The predominant peak around $1.8 \AA$ can be ascribed to Pt-C or Pt-N coordination ${ }^{20,31}$. The XANES also indicates an oxidized electronic structure of Pt (inset of Fig.2b). Further study shows that A-CoPt-C without $\mathrm{N}$ doping, as a control experiment, exhibits a high amount of metallic Pt-Pt coordination (Supplementary Fig.3). The corresponding $\mathrm{Pt}$ particles are also observed in A-CoPt-C by the TEM images in Supplementary Fig.4. These findings suggest that $\mathrm{N}$ plays a critical role in trapping single $\mathrm{Pt}$ atoms and a Pt-N-C coordination structure is highly possible.

Fig. $2 d$ shows the distribution of single metal atoms. Interestingly, these single metal atoms do not distribute uniformly on the carbon, but predominantly locate at the interstice of the graphitic layers. Fig. 2e is the bright field image focussing on the same area shown in Fig. $2 \mathrm{~d}$, clearly illustrating the opening of graphitic layers. Since amorphous carbon is less stable during the electrochemical activation and more easily oxidized ${ }^{32}$, we can deduce that those openings were created through the corrosion of partially existing amorphous carbon in the shell during the activation process, which is evidenced by increased graphitization degree of ACoPt-NC at $26.2^{\circ}$ in the XRD pattern (Supplementary Fig.1). With the removal of amorphous carbon forming the interstices, the cross-sections of the graphitic layers were exposed and distorted, thereby increasing the disorder of the lattice. The acidic solution subsequently permeated through these openings and dissolved the cobalt cores. Simultaneously, the Co and 
Pt ions near the interstices were captured by the exposure of lattice defects in the carbon with the assistance of the freshly generated dangling carbon and nitrogen bonds. These contribute to the atomic metal species being distributed locally at the graphitic layer openings. The statistical distribution ${ }^{11}$ of 40 pairs of two adjacent metal atoms (denoted as Me-Me, Me $=\mathrm{Co}$ or Pt) shows that the Me-Me distances are in the range from $0.2 \mathrm{~nm}$ to $0.5 \mathrm{~nm}$ with most in the interval of $0.25-0.29 \mathrm{~nm}$. This phenomenon provides the experimental basis for structures used in modelling, as discussed later.

Benefiting from fast Fourier Transformation (FFT) and inverse FFT, noise was filtered and the HAADF image of A-CoPt-NC with strong contrasts was gained from a less damaged region of interest (ROI). This is shown in Fig. $2 \mathrm{~g}$ with a clear view of the local coordination environment of the metal atoms. In Fig. 2h, the metal atoms are marked with purple cycles and the adjacent carbon/nitrogen atoms are linked. As a result, the configuration of the metal atoms (Me-\#1 and Me-\#2) can be identified. The structure observed in the STEM image is simulated in Fig. 2i. The same arrangement of the numbered atoms in Fig. $2 \mathrm{~h}$ are used to obtain insight into the structure-property correlation in A-CoPt-NC catalyst through computer simulations.

\section{Electrocatalytic ORR activity of A-CoPt-NC}



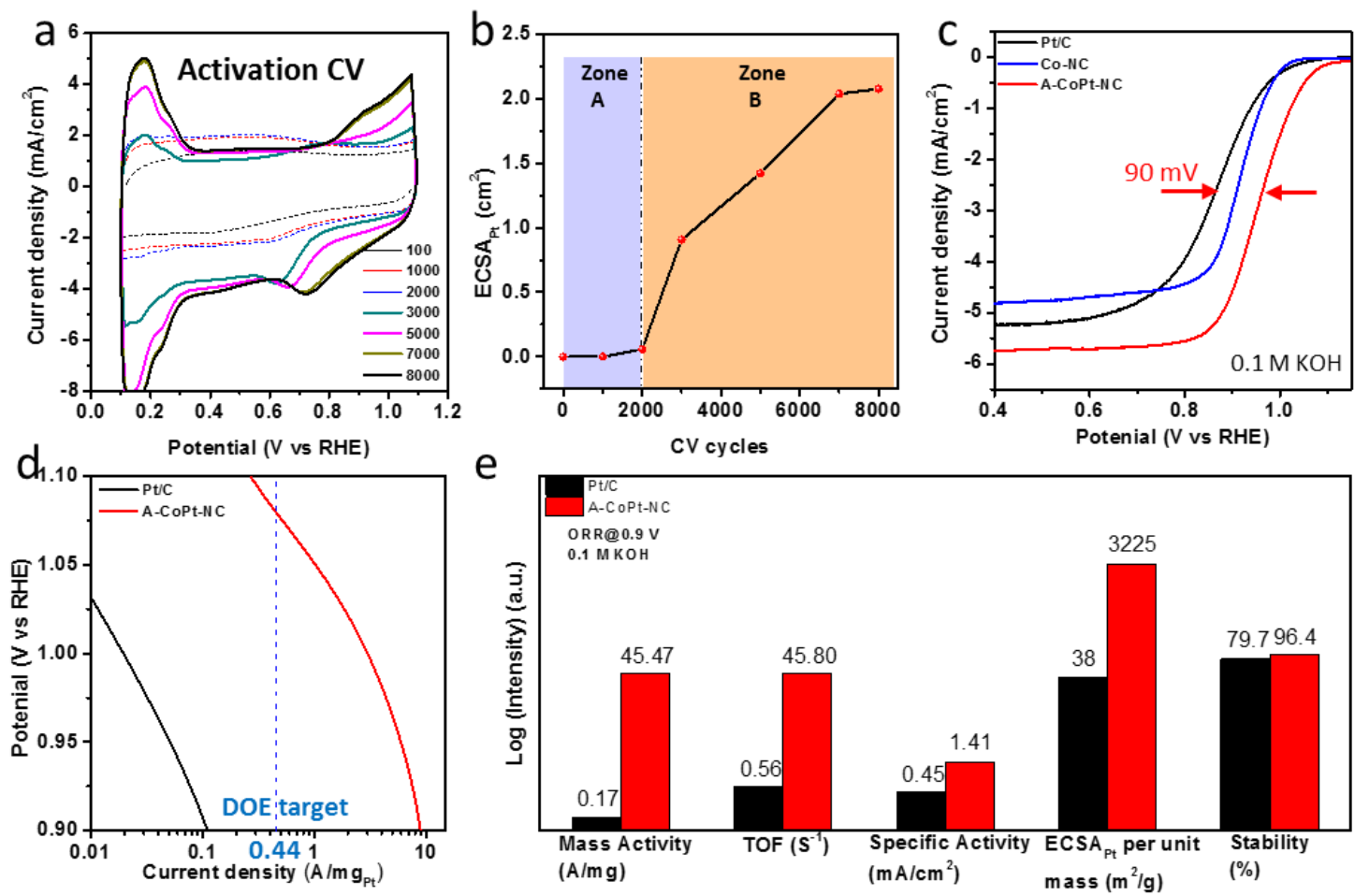

Figure 3 | Electrochemical oxygen reduction activities. a, Cyclic voltammetry (CV) curves after different activation cycles in $0.5 \mathrm{M} \mathrm{H}_{2} \mathrm{SO}_{4}$ electrolyte. $\mathbf{b}$, The evolution of the ECSAPt during activation. c, ORR linear sweep voltammetry (LSV) curves of CoNC, A-CoPt-NC and Pt/C in $0.1 \mathrm{M} \mathrm{KOH}$ electrolyte. d, Mass activity Tafel plot for ACoPt-NC and Pt/C. e, Comparison of mass activity, TOF, specific activity, ECSAPt per unit Pt mass and stability of the A-CoPt-NC and Pt/C for ORR.

The electrochemical activation was performed with $\mathrm{CV}$ cycles from $0.1 \mathrm{~V}$ to $1.1 \mathrm{~V}$ vs RHE in $0.5 \mathrm{M}$ sulphuric acid. Fig. 3a shows the evolution of the $\mathrm{CV}$ curves during activation. At the beginning of activation, no typical hydrogen adsorption or desorption peaks in the range of $0.1 \mathrm{~V}$ to $0.38 \mathrm{~V}$ (signifying the presence of $\mathrm{Pt}$ ) were observed. The hydrogen adsorption peak appeared after $2000 \mathrm{CV}$ cycles, indicating Pt started to load onto the carbon shell. The loading amount of Pt increased with the additional CV cycles until 7000 cycles. From 7000 to 8000 
cycles, the CV curves nearly overlapped demonstrating the saturation of Pt loading. The corresponding ECSA analysis of the Pt also reveals that in the first $2000 \mathrm{CV}$ cycles (zone A in Fig. 3b), there was little Pt loading on the shell (the ECSAPt is nearly zero) due to the rarity of adsorption sites in the carbon. During this stage, the amorphous carbon was oxidised and the graphitic shell cracked. With the continued oxidation, an increasing number of adsorption sites at the openings were generated and the Pt atoms were subsequently anchored (shown as zone B).

Linear sweep voltammetry (LSV) curves in $0.1 \mathrm{M} \mathrm{KOH}$ electrolyte (Supplementary Fig.5) shows the current density increased with the rotation rate from 400 to $2500 \mathrm{rpm}$, indicating a defined mass transfer controlled process. The Koutecky-Levich plot shown in the inset implies a $4 \mathrm{e}^{-}$transfer pathway for the ORR. The rotating ring-disk electrode (RRDE) voltammogram of A-CoPt-NC is also performed in $\mathrm{O}_{2}$-saturated $0.1 \mathrm{M} \mathrm{KOH}$ electrolyte at a rotation rate of $1600 \mathrm{rpm}$ to accurately determine the electron transfer number. Based on the ring and disk currents, the electron transfer number is calculated to be larger than 3.6 over the potential range from 0.4 to $1.0 \mathrm{~V}$ vs RHE, and the $\mathrm{H}_{2} \mathrm{O}_{2}$ yield remained below $17 \%$ (Supplementary Fig.6), indicating that 4e- transfer pathway is predominant in A-CoPt-NC for ORR in $0.1 \mathrm{M} \mathrm{KOH}$ electrolyte. The polarization curves in Fig. 3c show that the Co-NC exhibited higher half-wave potential $(0.92 \mathrm{~V}$ vs RHE) but lower limit current density (4.8 $\mathrm{mA} / \mathrm{cm}^{2}$ ) compared to those of commercial Pt/C catalyst, due to the metal-N-C catalysis ${ }^{33}$. Notably, the A-CoPt-NC exhibited much better performance than those of Co-NC and Pt/C as well as a robust stability (as shown in Supplementary Fig.7, after 4 hours reaction, A-CoPt-NC still retains the $96.4 \%$ of the initial activity whereas the commercial Pt/C catalyst only preserves 79.7\%). The half-wave potential of A-CoPt-NC is $0.96 \mathrm{~V}$ vs RHE, $90 \mathrm{mV}$ superior to that of Pt/C. The mass activity Tafel plot (Fig. 3d) shows that the A-CoPt-NC can deliver 20 times higher mass activity than the 2017 target set by the DOE (a current density of $0.44 \mathrm{~A} / \mathrm{mg}_{\mathrm{Pt}}$ at 
$0.90 \mathrm{~V}$, highlighted by blue dash line in Fig. 3d). The A-CoPt-NC can deliver the DOE targeted mass activity at $1.08 \mathrm{~V}$ vs RHE, thus reducing the overpotential by $0.18 \mathrm{~V}$. Impressively, the

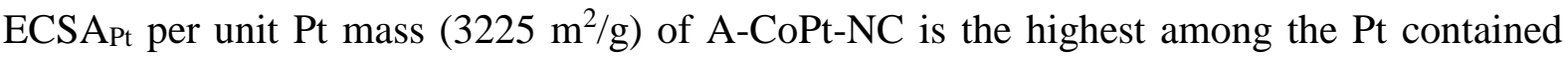
ORR catalysts reported so far (Fig. 3e), which is attributed to the high atom efficiency ${ }^{1,2,4}$. With this advantage, the A-CoPt-NC also presents a much higher mass activity of $45.47 \mathrm{~A} / \mathrm{mg}$ compared to those of Pt/C and other Pt contained catalysts (Supplementary Table 1). More importantly, the specific activity of A-CoPt-NC, which normalizes the performance to the ECSA, is 3-fold greater than that of $\mathrm{Pt} / \mathrm{C}$, indicating that the individual active site in $\mathrm{A}-\mathrm{CoPt}$ $\mathrm{NC}$ is more energetically favourable for ORR than that of Pt/C (Fig. 3e). As well as in the alkaline media, the A-CoPt-NC presents a very good ORR performance in the acidic electrolyte $\left(0.1 \mathrm{M} \mathrm{HClO}_{4}\right)$, with a mass activity 5.6 times higher than that of $\mathrm{Pt} / \mathrm{C}$ (Supplementary Fig.8).

\section{Catalytic mechanism of ORR in A-CoPt-NC}
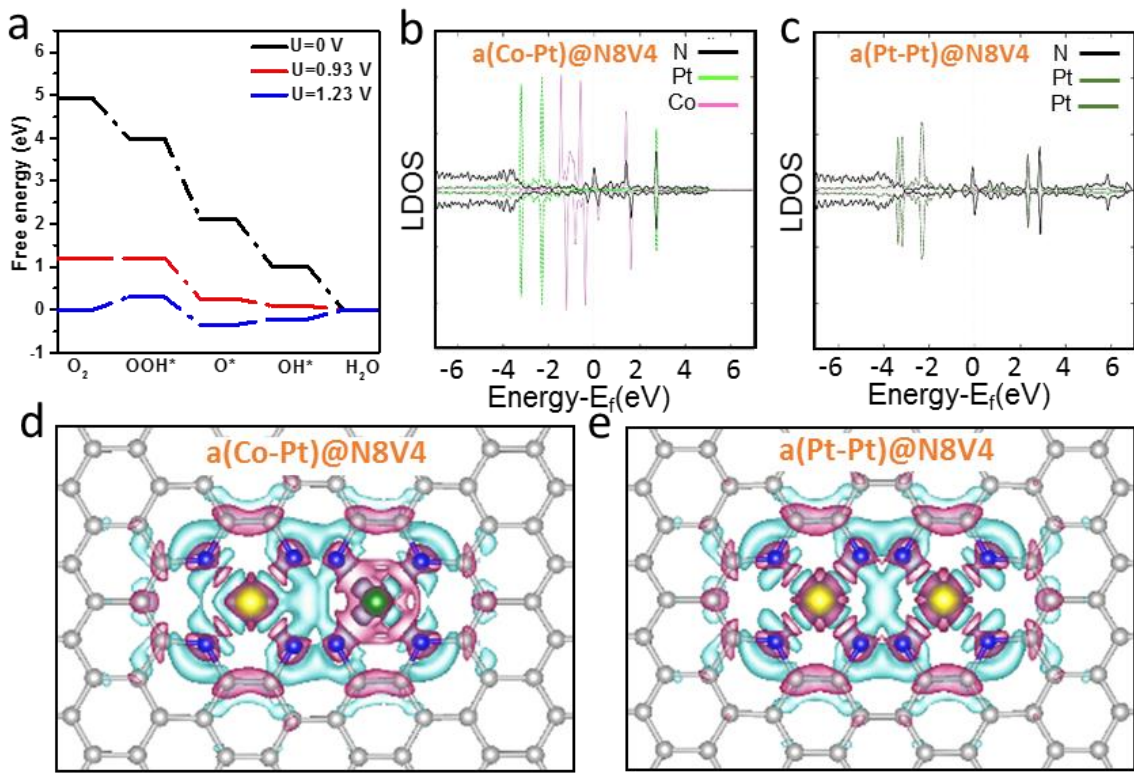

f

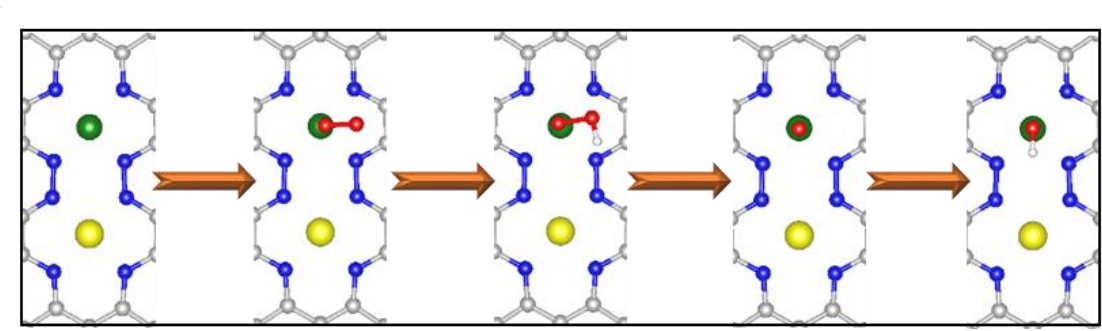


Figure 4 | Mechanistic study of ORR in A-CoPt-NC. a, The ORR free energy profiles of a(Co-Pt)@N8V4 at the equilibrium potential $(\mathrm{U}=1.23 \mathrm{~V})$, onset potential and zero potential. b,c, The local densities of states of a(Co-Pt)@N8V4 and a(Pt-Pt)@N8V4. d,e, The top view of the charge densities of a(Co-Pt)@N8V4 (d) and a(Pt-Pt)@N8V4 (e). Pink and aqua iso-surfaces with an isosurface level of $0.0025 e / a_{0}^{3}$ represent electron accumulation and depletion areas respectively. $\mathbf{f}$, An illustration of the ORR reaction pathway on a(Co-Pt)@N8V4.

According to the published literature, the atomic Pt based catalysts have a low selectivity for the $4 \mathrm{e}^{-}$transfer pathway for ORR (preferring to produce $\mathrm{H}_{2} \mathrm{O}_{2}$ rather than $\mathrm{H}_{2} \mathrm{O}$ ), due to the need of a synergistic effect from Pt sites within a desirable distance to break the O-O bond ${ }^{19-21}$. Therefore, we hypothesize that the synergetic effect of atomic $\mathrm{Pt}-\mathrm{Me}(\mathrm{Me}=\mathrm{Co} / \mathrm{Pt})$ coupling species at the carbon defects (denoted as a(Pt-Me)@ Defects) in A-CoPt-NC can enhance the selectivity of $4 \mathrm{e}^{-}$transfer pathway and the overall activity by modulating the electronic structure of metal atoms and altering the charge distribution at the coordination structures. To investigate the synergistic effect, we performed DFT calculations on five different coordination structures (each of them contains Pt-Pt and Co-Pt coordination as shown in Supplementary Fig.9), which were selected according to the analysis of the distribution of adjacent metal atomic interdistances (Fig. 2f) and STEM observations (Fig 2g-2i). The distances between the two adjacent metal atoms in these 5 models are in the range from $0.227 \mathrm{~nm}$ to $0.504 \mathrm{~nm}$, which are in accord with the experimentally measured distances (Fig. 2f). Notably, the structure $\mathrm{N} 8 \mathrm{~V} 4$ is directly observed from the STEM image in Fig. $2 \mathrm{~h}$. The binding energy of the atomic metals on the 5 models were calculated as shown in Supplementary Table 2. It is shown that atomic metals on N6V6 and N8V10 are not thermodynamically stable, thereby they are excluded for further calculations. Accordingly, six ORR energy profiles are obtained as shown 
in Supplementary Fig.10 and the corresponding onset potentials are summarized in Supplementary Table 3. The most energetically favourable configuration is a(Co-Pt)@N8V4 with a low overpotential of $0.30 \mathrm{~V}$. As shown in Fig. 4a, the first protonation step $\left(\mathrm{O}_{2} \rightarrow \mathrm{OOH}^{*}\right)$ determined the onset potential, because the magnitude of change in free energy $(0.3 \mathrm{eV})$ for this step is the largest at equilibrium. Meanwhile, the potential determine step for a(PtPt)@N8V4 is the same with a(Co-Pt)@N8V4 (Supplementary Fig.10), whereas requires an overpotential as large as $1.07 \mathrm{~V}$ (Supplementary Table 3). These results reveal that heterogeneous atomic metals (Co and $\mathrm{Pt}$ ) on N8V4 precede the homogenous atomic metals (sole Pt) on N8V4, due to the stronger binding effect between a(Co-Pt)@N8V4 and $\mathrm{O}_{2}$.

To investigate the underlying origin of the interactions between different active sites and the adsorbates, the density of the states of a(Co-Pt)@N8V4 and a(Pt-Pt)@N8V4 were simulated (Fig. $4 \mathrm{~b}$ and $4 \mathrm{c}$ ). Since the $d$ orbitals of noble/transition metal atoms and the $2 p$ orbitals of oxygen atoms participate in orbital coupling and form the new molecular orbitals during the adsorption, we concentrate on the states of $d$ orbitals of Pt and Co. According to the $d$ band centre theory proposed by Nørskov et al., the up-shifted $d$ orbital relative to the Fermi level will result in a strong binding between the catalyst and the adsorbate, and vice versa ${ }^{34}$. Here, the energy of the Co $3 d$ orbital in a(Co-Pt)@N8V4 is much closer to the Fermi level than that of the Pt $5 d$ orbital in a(Pt-Pt)@N8V4, indicating the strong binding between the a(CoPt)@N8V4 and oxygen. This difference in binding strength will further affect the ORR activities of these two active sites, which is supported by the energy profiles (Fig. 4a). In addition, the charge distribution patterns (Fig. 4d and 4e) show a strong electron accumulation (pink area) around the Co atom in a(Co-Pt)@N8V4, but weak electron accumulation or depletion around $\mathrm{Pt}$ in $\mathrm{a}(\mathrm{Pt}-\mathrm{Pt}) @ \mathrm{~N} 8 \mathrm{~V} 4$, which can be attributed to the asymmetric deployment of $\mathrm{Pt}$ and $\mathrm{Co}$ in a(Co-Pt)@N8V4, polarizing the surface charges near the active sites. The electrons near Co will enable $\mathrm{O}_{2}$ to be transformed to $\mathrm{H}_{2} \mathrm{O}$, thus enhancing the ORR 
performance. Fig. 4f shows the ORR reaction associated with the $4 \mathrm{e}^{-}$pathway on a(CoPt)@N8V4, which involves four protic hydrogen and electron transfer steps: (i) the adsorbed $\mathrm{O}_{2}$ transfers into $\mathrm{OOH}^{*}$; (ii) desorption of $\mathrm{H}_{2} \mathrm{O}$ and formation of $\mathrm{O}^{*}$; (iii) $\mathrm{OH}^{*}$ is formed and (iv) the $\mathrm{OH}^{*}$ further associates with a protic $\mathrm{H}$ and an electron to generate $\mathrm{H}_{2} \mathrm{O}$.

It is worth noting that the elementary steps from $\mathrm{O}_{2}$ to $\mathrm{O}^{*}$ can also proceed through a dissociative $4 \mathrm{e}^{-}$pathway, which is also energetically downhill in the energy profile (Supplementary Fig. 11) with an even lower overpotential of only $0.21 \mathrm{~V}$. In that case, the last $\mathrm{H}_{2} \mathrm{O}$ desorption step is the reaction determining step. The reaction mechanism for the dissociative $4 \mathrm{e}^{-}$pathway separates the oxygen bond in the first protonation step producing $\mathrm{O}^{*}$ and $\mathrm{OH}^{*}$ instead of forming $\mathrm{OOH}^{*}$. Thermodynamically, this pathway is favourable, but the $\mathrm{O}-$ $\mathrm{O}$ dissociation has a kinetic barrier with an activation energy of $0.56 \mathrm{eV}$. This is less than half the barrier on single Pt atoms on a simular substrate ${ }^{35}$, and could be further influenced due to solvent effects. Both these two pathways are different from that of IA-Pt catalysts ${ }^{21,35}$, which follow a $2 \mathrm{e}^{-}$pathway and has $\mathrm{H}_{2} \mathrm{O}_{2}$ as an intermediate product. Since O-O dissociation is the key step of the $4 \mathrm{e}^{-}$pathway, it is suggested that the A-CoPt-NC exhibits a different reaction pathway compared to the reported IA-Pt catalysts ${ }^{21,35}$. Here, we attribute the high $4 \mathrm{e}^{-}$pathway selectivity of A-CoPt-NC to the specific configuration structure of a(Co-Pt)@ defect and the synergistic effect between the atomic Co and Pt sites, which results in the up-shifting of the d orbital and the charge polarization on the active site (a(Co-Pt)@N8V4). These factors eventually alter the dissociation energy of $\mathrm{O}-\mathrm{O}$ bond and endow the $4 \mathrm{e}^{-} \mathrm{ORR}$ pathway in ACoPt-NC.

\section{Electrocatalytic HER activity of A-CoPt-NC in wide pH range}



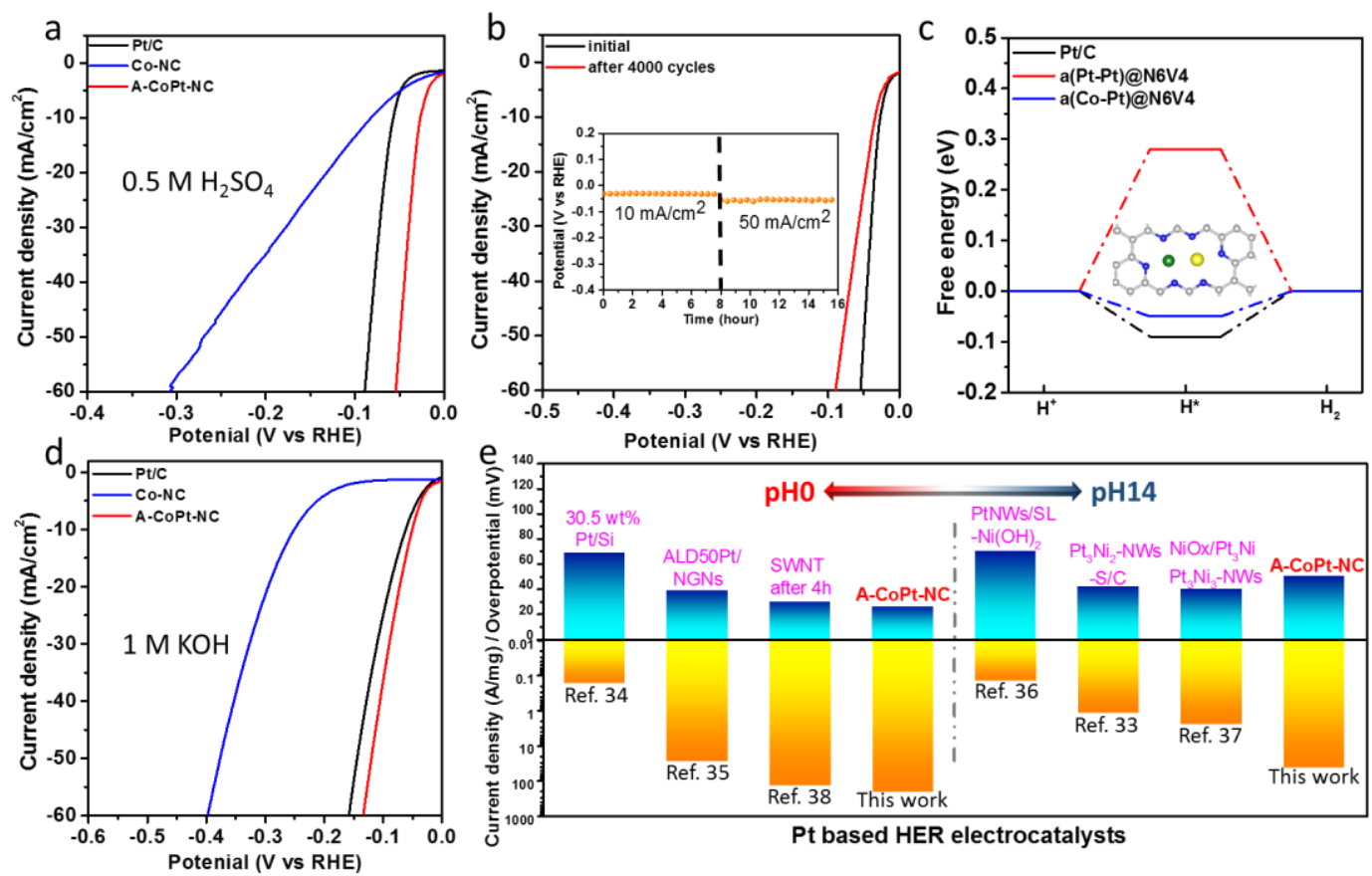

Figure 5 | Electrochemical hydrogen evolution activities. a, HER LSV curves of Co-NC, A-CoPt-NC and Pt/C in $0.5 \mathrm{M} \mathrm{H}_{2} \mathrm{SO}_{4}$ electrolyte. b, The durability test of ACoPt-NC for HER. The polarization curves were recorded initially and after $4000 \mathrm{CV}$ sweeps at a rate of $100 \mathrm{mV} / \mathrm{s}$. The inset contains chronopotentiometry curves with a current density of $10 \mathrm{~mA} / \mathrm{cm}^{2}$ and $50 \mathrm{~mA} / \mathrm{cm}^{2}$, respectively. c, The HER free energy profiles of the Pt/C, 2H/a(Pt-Pt)@N6V4 and 2H/a(Co-Pt)@N6V4. Top view of the model a(Co-Pt)@N6V4 are inset. d, HER LSV curves of Co-NC, A-CoPt-NC and Pt/C in $1 \mathrm{M} \mathrm{KOH}$ electrolyte. $\mathbf{e}$, The comparison of the overpotential needed to reach a current density of $10 \mathrm{~mA} / \mathrm{cm}^{2}$ (up) and the current density normalized to Pt mass at an overpotential of $70 \mathrm{mV}$ (down) of various HER catalysts in the wide $\mathrm{pH}$ range. The data were collected from ref. ${ }^{36-41}$.

As demonstrated above, electron accumulation on Co will benefit for the electrocatalytic reduction reactions, so it is natural to consider the A-CoPt-NC as an ideal catalyst for the HER. 
Fig. 5a shows the LSV curves of Co-NC, A-CoPt-NC and Pt/C in the $0.5 \mathrm{M} \mathrm{H}_{2} \mathrm{SO}_{4}$ solution. The overpotentials at a current density of $10 \mathrm{~mA} / \mathrm{cm}^{2}$ were measured to be $27 \mathrm{mV}$ for A-CoPt$\mathrm{NC}$ and $59 \mathrm{mV}$ for Pt/C, respectively (Supplementary Fig.12). A-CoPt-NC also exhibits a similar Tafel slope of $31 \mathrm{mV} / \mathrm{dec}$ to Pt/C, indicating the Volmer-Tafel pathway. Moreover, the durability evaluation (Fig. 5b) shows that after $4000 \mathrm{CV}$ cycles, only a slight decay can be observed in the LSV curve and the chronopotentiometry curves retain stable for 8 hours reaction at the current densities of $10 \mathrm{~mA} / \mathrm{cm}^{2}$ and $50 \mathrm{~mA} / \mathrm{cm}^{2}$, respectively, indicating good stability of the A-CoPt-NC during the long-term HER reaction in an acidic environment. To further investigate the reaction mechanism of A-CoPt-NC for HER, we determined the energy profiles using DFT calculations. As shown in Fig. 5c, the two optimized configurations with the lowest $\left|\Delta G_{H}\right|$ are a(Co-Pt)@N6V4 and a(Pt-Pt)@N6V4, differing from that in ORR process. A $\left|\Delta G_{H}\right|$ value of zero represents the ideal interacting energy between the adsorbed hydrogen and the catalyst. The value of $\left|\Delta G_{H}\right|$ for a(Co-Pt)@ N6V4 is $0.05 \mathrm{eV}$, lower than those of Pt/C and a(Pt-Pt)@N6V4. The local densities of states (Supplementary Fig.13) of a(CoPt)@N6V4 show an obvious higher state density than a(Pt-Pt)@N6V4 near the Fermi level, thereby increasing the coupling strength of the $\mathrm{H}^{*}$ and catalysts. Furthermore, the charge distribution patterns (Supplementary Fig.14) demonstrate that the a(Co-Pt)@N6V4 possesses higher electron density around Co atom than that around Pt atoms in a(Pt-Pt)@N6V4, implying the strong bonding capability with $\mathrm{H}^{*}$ at $\mathrm{Co}$ site. In desorption step of $\mathrm{H}^{*}$, for a(Co-Pt)@ N6V4, the coupling is not strong enough, while for $\mathrm{Pt} / \mathrm{C}$, the interactions are stronger and thus the desorption of the $\mathrm{H}_{2}$ produced requires more energy than for a(Co-Pt)@N6V4.

Considering that different HER devices in the realistic applications may be operated in a various range of $\mathrm{pH}$, a wide $\mathrm{pH}$ range tolerance is significant for the HER catalyst. Moreover, the wide $\mathrm{pH}$ range tolerance also endows the catalyst to be multifunctional with OER or/and ORR in their specific $\mathrm{pH}$ ranges. Fig. 5d indicates that A-CoPt-NC outperforms Pt/C in alkaline 
media for HER. Due to the ultralow loading of Pt $(0.16 \mathrm{wt} \%)$ in A-CoPt-NC, it can be a promising alternative to commercial $\mathrm{Pt} / \mathrm{C}$ catalyst and other Pt contained catalysts in the wide $\mathrm{pH}$ range of HER applications. Fig. 5e and Supplementary Table 4 show $\eta_{10}$ (the overpotential required to reach a current density of $10 \mathrm{~mA} / \mathrm{cm}^{2}$ ) and $J_{\mathrm{m}, 70}$ (activity normalized to Pt mass at an overpotential of $70 \mathrm{mV}$ ) of various reported catalysts and $\mathrm{A}-\mathrm{CoPt}-\mathrm{NC}$ in the wide $\mathrm{pH}$ range (A-CoPt-NC is from this work and the other data were collected from ref ${ }^{36-41}$ ). The A-CoPtNC exhibits the lowest $\eta_{10}$ in acidic media and a competitive $\eta_{10}$ in alkaline media for HER compared to other Pt based electrocatalysts. Considering the advantage of ultralow loading, the A-CoPt-NC achieves the highest $J_{\mathrm{m}, 70}$ in both acidic and alkaline media, which are 224 and 45 $\mathrm{A} / \mathrm{mg}$, respectively. Even in the neutral media, A-CoPt-NC still exhibits a comparable activity to Pt/C catalyst (Supplementary Fig.15), implying the A-CoPt-NC could be utilized over the full $\mathrm{pH}$ range from acid to alkaline. Supplementary Fig.16 and Supplementary Table 5 summarized the $\eta_{10}$ of all $\mathrm{pH}$ range functioned HER catalysts without Pt and the A-CoPt-NC in this work (the Pt content is extremely low, with a $\sim 0.0005 \mathrm{mg} / \mathrm{cm}^{2}$ loading). Compared to these reported catalysts, A-CoPt-NC presents the highest HER activities in acidic and alkaline media and comparable activity in neutral media.

\section{Conclusions}

Utilizing the strategy of the electrochemical activation, Co cores were removed from the stable $\mathrm{Co} / \mathrm{C}$ core-shell structures producing nitrogen doped defective carbons with atomic metal species. The activation process enables the removal of amorphous carbons, creating channels in graphitic carbon shells to allow ingress of acidic solvent and the gradual removal Co cores. Some of the atomic Co species were captured in the carbon shell. When using a Pt electrode during the activation, atomic Pt species from the dissolution in electrolyte can also be cocaptured by the N-doped carbon shell. Accordingly, a carbon-based catalyst decorated by co $\mathrm{Co} / \mathrm{Pt}$ at an atomic scale is synthesized. The resulting catalyst (denoted as A-CoPt-NC) only 
contains a small amount of Co $(\sim 1.72 \mathrm{wt} \%)$ and a very little Pt $(\sim 0.16 \mathrm{wt} \%)$, but shows extremely high activities for both ORR and HER. The ORR mass activity is as high as 267 times of the commercial $\mathrm{Pt} / \mathrm{C}$ and 20 times of the US DOE target at $0.90 \mathrm{~V}$ in alkaline. The catalyst also exhibits considerably high ORR activity in acid but requires further improvement, which is being considered in our further research. Moreover, the HER of this catalyst is much superior to the commercial Pt/C both in acid and alkaline media. DFT calculations suggested that the excellent electrocatalytic performance may originate from the charge redistribution and the $d$ orbital shift resulting from the synergetic effect of the atomic Pt and Co species in the specific coordination structure (a(Co-Pt)@N8V4 for ORR and a(Co-Pt)@N6V4 for HER). It is found that the atomic interaction of Pt-Co may be responsible for the high selectivity for $4 \mathrm{e}^{-}$ pathway in ORR, differing from the reported $2 \mathrm{e}^{-}$pathway in isolated atomic Pt-based catalysts. Thus, the appropriate coordination environment of atomic metal species by defect engineering is of importance to tune the corresponding electronic redistribution for electrocatalysis, calling for a re-thinking of accepted strategies for developing efficient electrochemical catalysts.

\section{Methods}

Synthesis of Co-MOF. In a typical synthesis, $0.2 \mathrm{~g} \mathrm{Co}\left(\mathrm{NO}_{3}\right)_{2} \cdot 6 \mathrm{H}_{2} \mathrm{O}, 0.21 \mathrm{~g}$ trimesic acid ( $\mathrm{H}_{3} \mathrm{BTC}$ ) and $0.02 \mathrm{~g} 4,4^{\prime}$-bipyridine were dissolved in the $8.47 \mathrm{~mL}$ dimethylformamide (DMF). Subsequently, $1 \mathrm{~mL}$ water and $7.18 \mathrm{~mL}$ diethylene glycol were added to the solution with 2 hours stirring to mix the components uniformly. Then the solution was kept at the temperature of $65^{\circ} \mathrm{C}$ for 48 hours. The Co-MOF was obtained after centrifugation.

Synthesis of A-CoPt-NC. A-CoPt-NC was prepared from Co-NC. Typically, the Co-MOF was mixed with dicyandiamide (mass ratio is $1: 16$ ) and annealed at $850{ }^{\circ} \mathrm{C}$ for 2 hours with a ramp rate of $4{ }^{\circ} \mathrm{C}$ under nitrogen atmosphere. Before calcining, the system was purged for two hours with nitrogen gas to ensure the removal of oxygen from the furnace. Then $4 \mathrm{mg}$ of the 
Co-NC was dispersed in the $1 \mathrm{~mL}$ ethanol/nafion $(200 \mu \mathrm{L} / 80 \mu \mathrm{L})$ solution for at least $30 \mathrm{~min}$ ultrasonication. $10 \mu \mathrm{L}$ of the mixture was dropped onto a polished glassy carbon electrode (4 $\mathrm{mm}$ in diameter).

Characterizations. Raman spectrum was recorded on a Renishaw InVia spectrometer with a model 100 Ramascope optical fibre instrument. X-ray photoelectron spectrum (XPS) data was collected from a Kratos Axis ULTRA X-ray photoelectron spectrometer, and the binding energy of the $\mathrm{C} 1 \mathrm{~s}$ peak at $284.8 \mathrm{eV}$ was used as an internal reference. Co and Pt K edge X-ray absorption fine structure (XAFS) data were collected at Hard X-ray micro analysis beamline (HXMA, 06ID). Transmission electron microscopy (TEM) images were collected from TECNAI 12 with acceleration voltages of $120 \mathrm{kV}$. Scanning transmission electron microscopyEnergy-dispersive X-ray spectroscopy (STEM-EDS) elemental mapping images were obtained from TECNAI G2 F20 with acceleration voltages of $200 \mathrm{kV}$. High angle annular dark field (HAADF) images and BF images are collected from probe-corrected JEOL ARM200F with acceleration voltages of $80 \mathrm{kV}$.

Electrochemical measurements. All the electrochemical tests were performed in a conventional three-electrode system at an electrochemical station (CHI 760E), using $\mathrm{Ag} / \mathrm{AgCl}$ (saturated $\mathrm{KCl}$ solution) electrode as the reference electrode, graphitic carbon rod as the counter electrode and glassy carbon (GC) electrode as the working electrode. All potentials were referred to the reversible hydrogen electrode (RHE) by following calculations: E (vs RHE) $=\mathrm{E}(\mathrm{vs} \mathrm{Ag} / \mathrm{AgCl})+0.197+0.059 \mathrm{pH} .4 \mathrm{mg}$ of sample and $80 \mu \mathrm{l}$ of $5 \mathrm{wt} \%$ Nafion solution were dispersed in $1 \mathrm{ml}$ of $4: 1 \mathrm{v} / \mathrm{v}$ water/ethanol by at least $60 \mathrm{~min}$ sonication to form a homogeneous solution. Then $5 \mu \mathrm{l}$ of the solution was loaded onto the GC electrode of $3 \mathrm{~mm}$ in diameter. The final loading for all catalysts and commercial $\mathrm{Pt} / \mathrm{C}$ electrocatalysts on the $\mathrm{GC}$ electrodes is about $0.262 \mathrm{mg} / \mathrm{cm}^{2}$. Linear sweep voltammetry with a scan rate of $5 \mathrm{mV} / \mathrm{s}$ was conducted in 
$1 \mathrm{M} \mathrm{KOH}$. Chronopotentiometry measurement $\left(\mathrm{j}=5 \mathrm{~mA} / \mathrm{cm}^{2}\right.$ and $\left.10 \mathrm{~mA} / \mathrm{cm}^{2}\right)$ was performed to evaluate the long-term stability.

Calculations. All calculations were performed with the Vienna Ab initio Simulation Package $(\mathrm{VASP})^{42}$ using density functional theory (DFT). The projector augmented-wave (PAW) ${ }^{43}$ method, the revised Perdew-Burke-Ernzerhof $(\mathrm{RPBE})^{44}$ functional and the dispersion correction by Grimme (DFT-D3) ${ }^{45}$ were used to describe the electronic interactions. Different structures were constructed to model the experimental systems to match the metal-metal distance. Pores were formed in a supercell of $8 \times 8$ graphene unit cells by removing carbon atoms. The different structures are illustrated and labelled in Supplementary Fig.8 (V - number of missing carbon atoms) and some carbon atoms at the edges were exchanged with nitrogen ( $\mathrm{N}$ - number of nitrogen atoms). The unit cell size was $19.74 \times 17.09 \times 20 \AA$ with a $2 \times 2 \times 1$ gamma-centred k-point grid and a cut off energy of $450 \mathrm{eV}$. These parameters were selected after carrying out preliminary tests. The free energies were calculated using a method similar to the approaches by Nørskov et al. ${ }^{46,47}$ (see supplementary information) using the zero point energy (ZPE) and change in entropy $\left(\Delta \mathrm{S}^{\circ}\right)$ for adsorption of the gases species on a zirconia surface $^{48}$. These results were used since a complete set of data was available and the results are expected to be insensitive to the surface.

\section{References}

1. Li MF, et al. Ultrafine jagged platinum nanowires enable ultrahigh mass activity for the oxygen reduction reaction. Science 354, 1414-1419 (2016).

2. He DP, et al. Amorphous nickel boride membrane on a platinum-nickel alloy surface for enhanced oxygen reduction reaction. Nature Communications 7, 12362 (2016).

3. Escudero-Escribano $M$, et al. Tuning the activity of Pt alloy electrocatalysts by means of the lanthanide contraction. Science 352, 73-76 (2016). 
4. $\quad \mathrm{Bu} \mathrm{LZ}$, et al. Biaxially strained PtPb/Pt core/shell nanoplate boosts oxygen reduction catalysis. Science 354, 1410-1414 (2016).

5. Huang $\mathrm{XQ}$, et al. High-performance transition metal-doped Pt3Ni octahedra for oxygen reduction reaction. Science 348, 1230-1234 (2015).

6. Chen Z, Ye S, Wilson AR, Ha Y-C, Wiley BJ. Optically transparent hydrogen evolution catalysts made from networks of copper-platinum core-shell nanowires. Energy \& Environmental Science 7, 1461-1467 (2014).

7. Sheng W, Zhuang Z, Gao M, Zheng J, Chen JG, Yan Y. Correlating hydrogen oxidation and evolution activity on platinum at different $\mathrm{pH}$ with measured hydrogen binding energy. Nature communications 6, 6848 (2015).

8. Roduner E. Size matters: why nanomaterials are different. Chemical Society Reviews 35, 583592 (2006).

9. Li L, et al. Investigation of catalytic finite-size-effects of platinum metal clusters. The journal of physical chemistry letters 4, 222-226 (2012).

10. Qiao BT, et al. Single-atom catalysis of $\mathrm{CO}$ oxidation using Pt-1/FeOx. Nat Chem 3, 634-641 (2011).

11. Moses-DeBusk M, et al. CO Oxidation on Supported Single Pt Atoms: Experimental and ab Initio Density Functional Studies of CO Interaction with Pt Atom on theta-Al2O3(010) Surface. J Am Chem Soc 135, 12634-12645 (2013).

12. Qiao B, et al. Highly efficient catalysis of preferential oxidation of $\mathrm{CO}$ in $\mathrm{H} 2$-rich stream by gold single-atom catalysts. ACS Catal 5, 6249-6254 (2015).

13. Yang $\mathrm{M}$, et al. Catalytically active $\mathrm{Au}-\mathrm{O}(\mathrm{OH})(\mathrm{x})$-species stabilized by alkali ions on zeolites and mesoporous oxides. Science 346, 1498-1501 (2014).

14. Lin J, et al. Remarkable Performance of Ir-1/FeOx Single-Atom Catalyst in Water Gas Shift Reaction. J Am Chem Soc 135, 15314-15317 (2013).

15. Yin PQ, et al. Single Cobalt Atoms with Precise N-Coordination as Superior Oxygen Reduction Reaction Catalysts. Angew Chem Int Ed 55, 10800-10805 (2016).

16. Fan LL, et al. Atomically isolated nickel species anchored on graphitized carbon for efficient hydrogen evolution electrocatalysis. Nat Commun 7, 10667 (2016). 
17. Qiu HJ, et al. Nanoporous Graphene with Single-Atom Nickel Dopants: An Efficient and Stable Catalyst for Electrochemical Hydrogen Production. Angew Chem Int Ed 54, 14031-14035 (2015).

18. Shen $\mathrm{H}$, et al. Atomically FeN 2 moieties dispersed on mesoporous carbon: A new atomic catalyst for efficient oxygen reduction catalysis. Nano Energy 35, 9-16 (2017).

19. Yang S, Tak YJ, Kim J, Soon A, Lee H. Support Effects in Single-Atom Platinum Catalysts for Electrochemical Oxygen Reduction. ACS Catal 7, 1301-1307 (2016).

20. Yang S, Kim J, Tak YJ, Soon A, Lee H. Single - Atom Catalyst of Platinum Supported on Titanium Nitride for Selective Electrochemical Reactions. Angew Chem Int Ed 55, 2058-2062 (2016).

21. Choi $\mathrm{CH}$, et al. Tuning selectivity of electrochemical reactions by atomically dispersed platinum catalyst. Nat Commun 7, 10922 (2016).

22. Jia $Y$, et al. Defect Graphene as a Trifunctional Catalyst for Electrochemical Reactions. Adv Mater 28, 9532-9538 (2016).

23. Tang $\mathrm{C}$, Wang $\mathrm{B}$, Wang $\mathrm{H}-\mathrm{F}$, Zhang Q. Defect Engineering toward Atomic $\mathrm{Co}-\mathrm{Nx}-\mathrm{C}$ in Hierarchical Graphene for Rechargeable Flexible Solid Zn-Air Batteries. Advanced Materials, 1703185.

24. Zhang L, et al. Defects on graphene trapping atomic Ni species for hydrogen and oxygen evolution reactions. Chem, (2018).

25. Xiao P, Ge X, Wang H, Liu Z, Fisher A, Wang X. Novel molybdenum carbide-tungsten carbide composite nanowires and their electrochemical activation for efficient and stable hydrogen evolution. Advanced Functional Materials 25, 1520-1526 (2015).

26. Das RK, et al. Extraordinary Hydrogen Evolution and Oxidation Reaction Activity from Carbon Nanotubes and Graphitic Carbons. Acs Nano 8, 8447-8456 (2014).

27. Fei $\mathrm{H}$, et al. Atomic cobalt on nitrogen-doped graphene for hydrogen generation. Nat Commun 6, 8668 (2015).

28. Zitolo A, et al. Identification of catalytic sites for oxygen reduction in iron- and nitrogen-doped graphene materials. Nature Materials 14, 937-942 (2015).

29. Liu W, et al. Single-atom dispersed Co-N-C catalyst: structure identification and performance for hydrogenative coupling of nitroarenes. Chemical Science 7, 5758-5764 (2016).

30. Alves MCM, Dodelet JP, Guay D, Ladouceur M, Tourillon G. Origin of the electrocatalytic properties for oxygen reduction of some heat-treated polyacrylonitrile and phthalocyanine 
cobalt compounds adsorbed on carbon black as probed by electrochemistry and x-ray absorption spectroscopy. The Journal of Physical Chemistry 96, 10898-10905 (1992).

31. Li XG, et al. Single-Atom Pt as Co-Catalyst for Enhanced Photocatalytic H-2 Evolution. Advanced Materials 28, 2427-2431 (2016).

32. Li L, Xing Y. Electrochemical durability of carbon nanotubes in noncatalyzed and catalyzed oxidations. Journal of the Electrochemical Society 153, A1823-A1828 (2006).

33. Varnell JA, et al. Identification of carbon-encapsulated iron nanoparticles as active species in non-precious metal oxygen reduction catalysts. Nature Communications 7, 12582 (2016).

34. Hammer B, Nørskov JK. Theoretical surface science and catalysis-calculations and concepts. Advances in catalysis 45, 71-129 (2000).

35. Liu S, Huang S. Theoretical insights into the activation of $\mathrm{O} 2$ by Pt single atom and Pt4 nanocluster on functionalized graphene support: Critical role of Pt positive polarized charges. Carbon 115, 11-17 (2017).

36. Wang PT, et al. Precise tuning in platinum-nickel/ nickel sulfide interface nanowires for synergistic hydrogen evolution catalysis. Nature Communications 8, 14580 (2017).

37. Zhu LL, et al. A rhodium/silicon co-electrocatalyst design concept to surpass platinum hydrogen evolution activity at high overpotentials. Nature Communications 7, 12272 (2016).

38. Cheng NC, et al. Platinum single-atom and cluster catalysis of the hydrogen evolution reaction. Nature Communications 7, 13638 (2016).

39. Yin $\mathrm{HJ}$, et al. Ultrathin platinum nanowires grown on single-layered nickel hydroxide with high hydrogen evolution activity. Nature Communications 6, 6430 (2015).

40. Wang PT, Jiang KZ, Wang GM, Yao JL, Huang XQ. Phase and Interface Engineering of PlatinumNickel Nanowires for Efficient Electrochemical Hydrogen Evolution. Angewandte ChemieInternational Edition 55, 12859-12863 (2016).

41. Tavakkoli $\mathrm{M}$, et al. Electrochemical Activation of Single-Walled Carbon Nanotubes with Pseudo-Atomic-Scale Platinum for the Hydrogen Evolution Reaction. ACS Catalysis 7, 31213130 (2017).

42. Kresse G, Furthmüller J. Efficient iterative schemes for ab initio total-energy calculations using a plane-wave basis set. Physical review B 54, 11169 (1996).

43. Blöchl PE. Projector augmented-wave method. Physical review B 50, 17953 (1994). 
44. Hammer B, Hansen LB, Nørskov JK. Improved adsorption energetics within density-functional theory using revised Perdew-Burke-Ernzerhof functionals. Physical Review B 59, 7413 (1999).

45. Grimme S, Antony J, Ehrlich S, Krieg H. A consistent and accurate ab initio parametrization of density functional dispersion correction (DFT-D) for the 94 elements H-Pu. The Journal of chemical physics 132, 154104 (2010).

46. Nørskov JK, et al. Origin of the overpotential for oxygen reduction at a fuel-cell cathode. The Journal of Physical Chemistry B 108, 17886-17892 (2004).

47. Nørskov JK, et al. Trends in the exchange current for hydrogen evolution. Journal of The Electrochemical Society 152, J23-J26 (2005).

48. Wang $G$, et al. A first-principle study of oxygen reduction reaction on monoclinic zirconia (11),(01) and (110) surfaces. Catalysis Communications 69, 16-19 (2015).

\section{Acknowledgements}

The authors thank the financial support from Australia Research Council (ARC DP170103317). Y.J. also thanks ARC Discovery Early Career Researcher Award (ARC DE180101030), the Griffith University Postdoctoral and Research Fellowship and Griffith University New Research Grant. The authors would like to thank the Australian National Fabrication Facility (ANFF) - Materials node, University of Wollongong (UOW) Electron Microscopy Centre (EMC) and Shanghai Synchrotron Radiation Facility for equipment access. J.M.T.A.F. thanks the University of Queensland for the UQI scholarship supporting her Ph.D. We acknowledge access to the computational resources of the NCI National Facility at the Australian National University through the National Computational Merit Allocation Scheme supported by the Australian Government; and support from the Queensland Cyber Infrastructure Foundation (QCIF) and the University of Queensland Research Computing Centre.

\section{Author contributions}

X.Y. conceived and designed the project. X.Y. and Y.J. supervised the project. L.Z. prepared the samples and did the electrocatalytic performances test. L.Z., X.Y., W.X., X.W., S.F. and J.C. performed the characterizations including XRD, XPS, TEM, XAS and so on. J.M.T.A.F., M.H. and D.J.S. performed the DFT calculations. L.Z., Y.J., X.Y. and J.M.T.A.F. wrote the manuscript. L.Z. and J.M.T.A.F. contributed equally to this work. All authors discussed the results and commented on the manuscript.

\section{Additional information}

Supplementary information is available for this paper. Reprints and permissions information is available at www.nature.com/reprints. Correspondence and requests for materials should be addressed to Y.J. and X.Y. 


\section{Competing interests}

The authors declare no competing financial interests. 